\title{
Classification with binary gene expressions
}

\author{
Salih Tuna, Mahesan Niranjan ${ }^{1}$
}

${ }^{1}$ School of Electronics and Computer Science, University of Southampton, Southampton, UK.

Email: $\underline{\text { mn@ecs.soton.ac.uk }}$

Received 30 March 2008; revised 25 May 2009; accepted 3 June 2009.

\section{ABSTRACT}

Microarray gene expression measurements are reported, used and archived usually to high numerical precision. However, properties of mRNA molecules, such as their low stability and availability in small copy numbers, and the fact that measurements correspond to a population of cells, rather than a single cell, makes high precision meaningless. Recent work shows that reducing measurement precision leads to very little loss of information, right down to binary levels. In this paper we show how properties of binary spaces can be useful in making inferences from microarray data. In particular, we use the Tanimoto similarity metric for binary vectors, which has been used effectively in the Chemoinformatics literature for retrieving chemical compounds with certain functional properties. This measure, when incorporated in a kernel framework, helps recover any information lost by quantization. By implementing a spectral clustering framework, we further show that a second reason for high performance from the Tanimoto metric can be traced back to a hitherto unnoticed systematic variability in array data: Probe level uncertainties are systematically lower for arrays with large numbers of expressed genes. While we offer no molecular level explanation for this systematic variability, that it could be exploited in a suitable similarity metric is a useful observation in itself. We further show preliminary results that working with binary data considerably reduces variability in the results across choice of algorithms in the pre-processing stages of microarray analysis.

Keywords: Microarray Gene Expression; Binary Gene Expressions; High Numerical Precision; mRNA Molecules

\section{INTRODUCTION}

It is anecdotally known and has been formally established recently that gene expression measurements archived in microarray repositories are reported to a far higher numerical precision than is supported by the underlying biology of the measurement environment. Here, precision refers to the difference between representing the mRNA abundance, or relative abundance, of a gene to several decimal places (e.g. 2.4601) and retaining only the binary information as to whether the gene is expressed or not. Shmulevich and Zhang [1] recommend that gene expressions should be quantized to binary precision and Hamming distance between signatures used as distance metric in solving class prediction problems. Their starting point in defining binary expressions is a "notion of similarity used by biologists when comparing gene expressions from different samples... counting the number of genes that show significant differential expression”. From this premise, they give an algorithm for binarizing gene expressions and show that a multi dimensional scaling (MDS) projection of the data separates different types of tumors. More recently, Zilliox and Irizarry [2] introduce the concept of gene expression "barcodes", which are essentially binary representations of transcriptomes, and present impressive results on predicting tissue types. These authors take a very different approach in that they scan through a very large number of archived datasets of a particular array type to construct barcodes. Genes that are frequently expressed across the whole ensemble are set to be $\mathrm{ON}$ and the others set OFF. In our own recent work [3], we showed that progressive quantization of gene expression measurements, right down to binary levels, loses very little information as far as the quality of inference is concerned. We were able to demonstrate this on a range of different inference problems including classification, cluster analysis, determination of genes that are periodically expressed and the analysis of developmental time course data.

Why would we be interested in low precision, or binary, representations? The initial motivation comes from the underlying biology. mRNA is only available in very 
small quantities in cells and are extracted from a population of cells rather than from a single cell. Further, the process of microarray hybridization itself is a stochastic one, the effect of which is pronounced when small numbers of molecules are involved. All these reasons put together make one sceptical about high precision representations of the transcriptome, i.e. the signal available may only be reliable to low precision. Critical appraisals of microarray technology, while recognising good reproducibility of technical replicates, often identifies large variations with respect to biological replicates. One such survey by Draghici et al. [4] concludes:

"...the existence and direction of gene expression changes can be reliably detected for the majority of genes. However, accurate measurements of absolute expression levels and the reliable detection of low abundance genes are currently beyond the reach of microarray technology.”

Artificially inflated precision can potentially hurt. A plethora of sophisticated inference methods (e.g. Bayesian inference) have been applied to microarray data. Algorithmic complexity of such models is generally derived from how well noise is captured. High precision gives the illusion of complex noise structures leading to the use of such algorithms. If the data were far simpler, one would impose a far higher sense of parsimony in model selection. Simple classification rules offering good performance (e.g. the top scoring pairs of genes approach of Geman et al. [5]) on some problems also bears testimony to this point. Motivated by the above, we ask the following research question: If transcriptome can be represented at low precision, binary for instance, can we take advantage of properties of high dimensional binary spaces to achieve increased classification performance? We show that this is indeed the case, by use of a particular similarity metric between high dimensional binary vectors, the so called Tanimoto metric. Following experiences seen in the chemoinformatics literature, we embed this similarity metric in a kernel discriminant framework (support vector machines-SVM) and show that very high classification accuracies are obtainable with binary representation of expression profiles. We offer explanations for why such increased performances can be achieved, and attribute this to two reasons: a) the training of class boundaries that happen in SVMs, and b) a hitherto unnoticed probe level uncertainty in microarray data.

Finally, the analysis of microarray data goes through a number of stages of processing steps: background intensity correction, within array normalization, between array normalization and algorithms for detecting differentially expressed genes. A user has a choice of several algorithms at each of these steps and a very large choice if we consider combinations of available algorithms. A particular appeal of working with binarized representations, as shown by preliminary results in this paper, is that the algorithmic variability in inference is drastically reduced without compromising the quality of inference.

\section{RESULTS}

\subsection{Classification}

Table 1 compares classification performances of several classifiers on six microarray class prediction datasets. In all cases the accuracies are averaged over 25 random partitions of the data into training and test sets, and standard deviations in performance across these partitions is also given. In all the different problems we checked to ensure that our implementation of the linear SVM classifier acting on raw data performed as well as the results quoted in the original publication or some other publication that used the dataset, thus confirming the correctness of our implementation. Note that in all the tasks considered, comparing data represented at raw and binary precisions and classifying with linear SVMs, we note that binarising the data has not lost much discrimination. In fact in some of the tasks binarization has actually improved performance. Secondly, in half the tasks considered, the use of Tanimoto kernel SVM improves the results of binarized classification. Where there is not an improvement, the method is at least as good as a linear SVM on binarized data.

Our simulations also show that in all the tasks considered the distance to template methods perform significantly worse than the corresponding kernel methods. This is true both for templates set as centroids and for centroids positioned optimally by genetic search. In two of the four datasets considered, optimization of templates quickly led to overtraining, resulting in classifiers whose performance on test data (entries in Table 1) were worse than their initial values (which were the performances with templates at centroids). In the genetic optimization, we also found that the local search by mutation was the dominant contributor, showing that the solution to the optimized distance based classifier was in the vicinity of the centroids. Cross-over operations nearly always produced far worse solutions and were quickly abandoned. To explore this further, in addition to the centroids, we included noisy templates into the search algorithm, but found no improvement.

\subsection{Clustering}

Figure 1 shows the eigenvector obtained in spectral clustering for the widely studied ALL/AML problem [11], computed in three different ways: raw and binarized data with negative exponential of Euclidean distance as similarity, and binarized data with Tanimoto similarity. The scatter clearly shows cluster separation along the components of the eigenvector. This is also 
reflected in the Fisher scores between clusters and the corresponding classification errors which are shown in Table 2, (columns 4 and 5), where except in one of the datasets, there is improvement in the cluster tightness when Tanimoto similarity is applied. Similarly, in all but one of the tasks, the resulting classification error rates are also lower for the Tanimoto metric.

The final column in Table 2 shows classification error rates arising from spectral clustering when the microarray profile consists of a filtered subset of genes. In each task we ranked the genes according to their Fisher scores of discriminating power taken one at a time, precisely the same way as done by Golub et al. [11], and report best performing subsets. The difference between the different distance metrics with subsets of genes is shown in Figure 2 for four of the tasks. We see that the use of Tanimoto similarity leads to better separated clusters in general. Further the better separated clusters also lead to better discrimination. We emphasize that the clustering here is done without the use of class labels, and it is to verify how good the clusters are that we use this information. Thus as expected note the accuracies much lower than when the problem is formulated as a classification problem in the first place.

Table 1. Comparison of classification with different types of kernels for SVM.

\begin{tabular}{|c|c|c|c|}
\hline Dataset & Data type & Method & Accuracy \\
\hline \multirow{5}{*}{ West et al. [6] } & Raw-Binary & Linear-SVM & $0.83 \pm 0.10$ \\
\hline & Binary & Linear-SVM & $0.86 \pm 0.08$ \\
\hline & Binary & Tanimoto-SVM & $0.87 \pm 0.08$ \\
\hline & Binary & Distance-to-class mean & $0.79 \pm 0.08$ \\
\hline & Binary & Distance-to-optimized template & $0.77 \pm 0.11$ \\
\hline \multirow{5}{*}{ Huang et al. [7] } & Raw-Binary & Linear-SVM & $0.63 \pm 0.12$ \\
\hline & Binary & Linear-SVM & $0.67 \pm 0.08$ \\
\hline & Binary & Tanimoto-SVM & $0.67 \pm 0.10$ \\
\hline & Binary & Distance-to-class mean & $0.60 \pm 0.11$ \\
\hline & Binary & Distance-to-optimized template & $0.66 \pm 0.11$ \\
\hline \multirow{5}{*}{ Gordon et al. [8] } & Raw-Binary & Linear-SVM & $0.99 \pm 0.01$ \\
\hline & Binary & Linear-SVM & $0.96 \pm 0.03$ \\
\hline & Binary & Tanimoto-SVM & $0.99 \pm 0.01$ \\
\hline & Binary & Distance-to-class mean & $0.88 \pm 0.07$ \\
\hline & Binary & Distance-to-optimized template & $0.90 \pm 0.07$ \\
\hline \multirow{5}{*}{ Brown et al. [9] } & Raw-Binary & Linear-SVM & $0.99 \pm 0.01$ \\
\hline & Binary & Linear-SVM & $0.98 \pm 0.01$ \\
\hline & Binary & Tanimoto-SVM & $0.98 \pm 0.01$ \\
\hline & Binary & Distance-to-class mean & $0.67 \pm 0.02$ \\
\hline & Binary & Distance-to-optimized template & $0.75 \pm 0.03$ \\
\hline \multirow{5}{*}{ Alon et al. [10] } & Raw-Binary & Linear-SVM & $0.78 \pm 0.11$ \\
\hline & Binary & Linear-SVM & $0.82 \pm 0.07$ \\
\hline & Binary & Tanimoto-SVM & $0.84 \pm 0.03$ \\
\hline & Binary & Distance-to-class mean & $0.80 \pm 0.07$ \\
\hline & Binary & Distance-to-optimized template & $0.72 \pm 0.10$ \\
\hline \multirow{5}{*}{ Golub et al [11]. } & Raw-Binary & Linear-SVM & $0.96 \pm 0.05$ \\
\hline & Binary & Linear-SVM & $0.95 \pm 0.03$ \\
\hline & Binary & Tanimoto-SVM & $0.96 \pm 0.04$ \\
\hline & Binary & Distance-to-class mean & $0.94 \pm 0.02$ \\
\hline & Binary & Distance-to-optimized template & $0.92 \pm 0.09$ \\
\hline
\end{tabular}




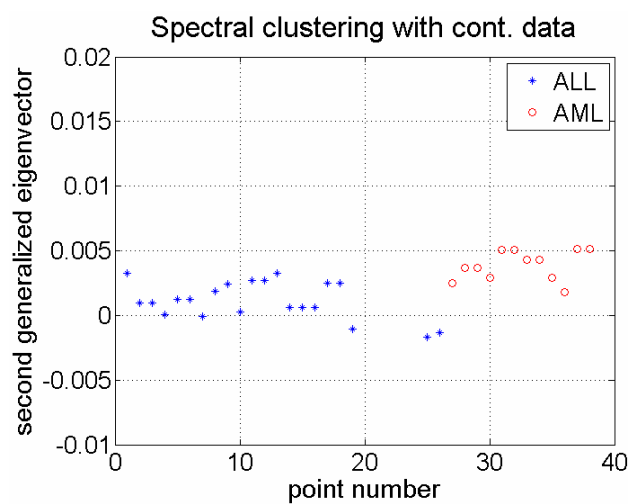

(a)

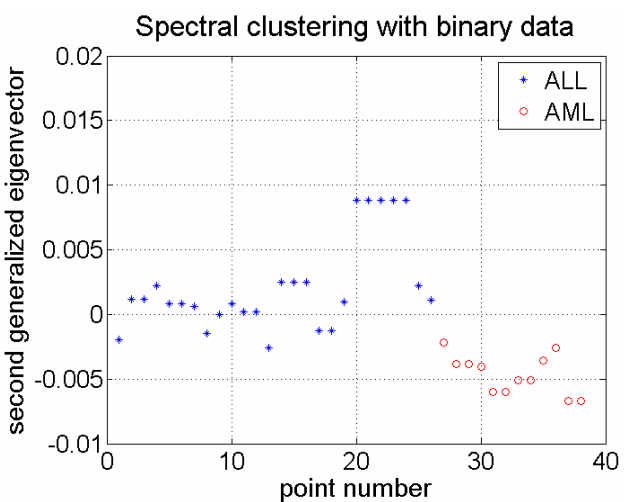

(b)

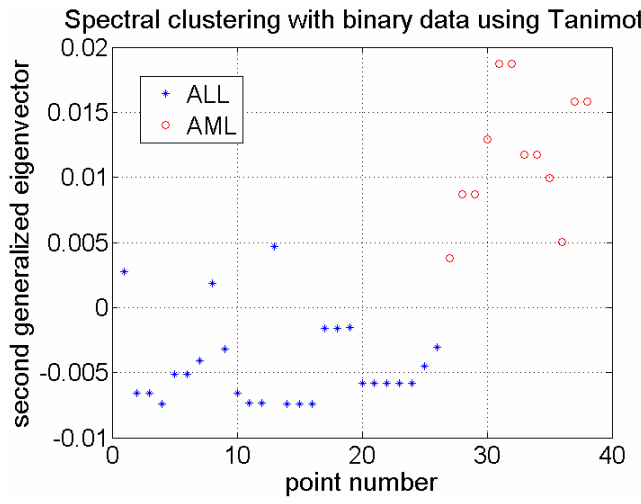

(c)

Figure 1. Figures showing spectral clustering results for different type of metrics. In (a) spectral clustering is applied to continuous data by using Euclidean distance, in (b) binary data is used with Euclidean distance and in (c) binary data is used with Tanimoto coefficient for spectral clustering. Data from [11].

Table 2. Comparison of spectral clustering results by using Tanimoto and Euclidean distance with Fisher score and error rates.

\begin{tabular}{|c|c|c|c|c|c|}
\hline Dataset & Data type & Distance metrics & Fisher score & Error rate & $\begin{array}{c}\text { Error rate } \\
\text { (best subset of genes) }\end{array}$ \\
\hline \multirow{3}{*}{ Simulated data } & Raw & Euclidean & $2.47 \pm 0.50$ & $0.14 \pm 0.08$ & \\
\hline & Binary & Euclidean & $0.47 \pm 0.49$ & $0.33 \pm 0.02$ & \\
\hline & Binary & Tanimoto & $0.66 \pm 0.21$ & $0.21 \pm 0.10$ & \\
\hline \multirow{3}{*}{ Golub et al. [11] } & Raw & Euclidean & $0.98 \pm 0.41$ & $0.32 \pm 0.23$ & $0.05 \pm 0.11$ \\
\hline & Binary & Euclidean & $1.01 \pm 0.43$ & $0.10 \pm 0.08$ & $0.02 \pm 0.04$ \\
\hline & Binary & Tanimoto & $1.49 \pm 0.42$ & $0.05 \pm 0.05$ & $0.004 \pm 0.02$ \\
\hline \multirow{3}{*}{ Huang et al. [7] } & Raw & Euclidean & $0.35 \pm 0.22$ & $0.21 \pm 0.05$ & $0.04 \pm 0.05$ \\
\hline & Binary & Euclidean & $0.37 \pm 0.18$ & $0.22 \pm 0.05$ & $0.03 \pm 0.05$ \\
\hline & Binary & Tanimoto & $0.33 \pm 0.17$ & $0.21 \pm 0.05$ & $0.02 \pm 0.04$ \\
\hline \multirow{3}{*}{ West et al. [6] } & Raw & Euclidean & $0.35 \pm 0.04$ & $0.45 \pm 0.06$ & $0.45 \pm 0.06$ \\
\hline & Binary & Euclidean & $0.30 \pm 0.18$ & $0.33 \pm 0.08$ & $0.21 \pm 0.15$ \\
\hline & Binary & Tanimoto & $0.35 \pm 0.24$ & $0.28 \pm 0.09$ & $0.11 \pm 0.07$ \\
\hline \multirow{3}{*}{ Gordon et al. [8] } & Raw & Euclidean & $0.21 \pm 0.07$ & $0.17 \pm 0.03$ & $0.16 \pm 0.03$ \\
\hline & Binary & Euclidean & $0.41 \pm 0.19$ & $0.13 \pm 0.02$ & $0.09 \pm 0.03$ \\
\hline & Binary & Tanimoto & $0.52 \pm 0.19$ & $0.12 \pm 0.02$ & $0.08 \pm 0.02$ \\
\hline
\end{tabular}




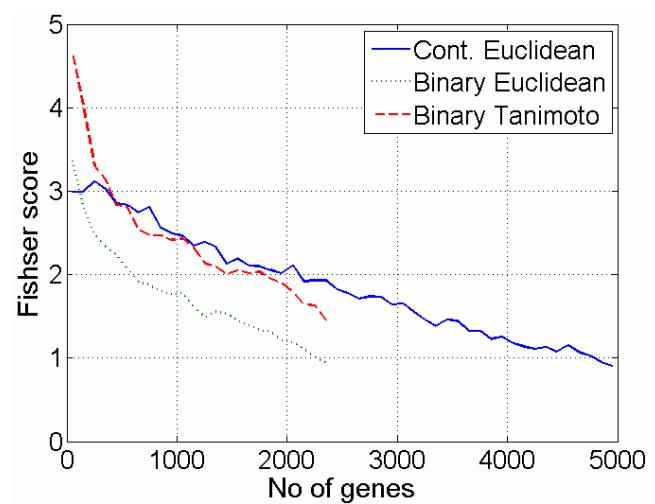

(a)

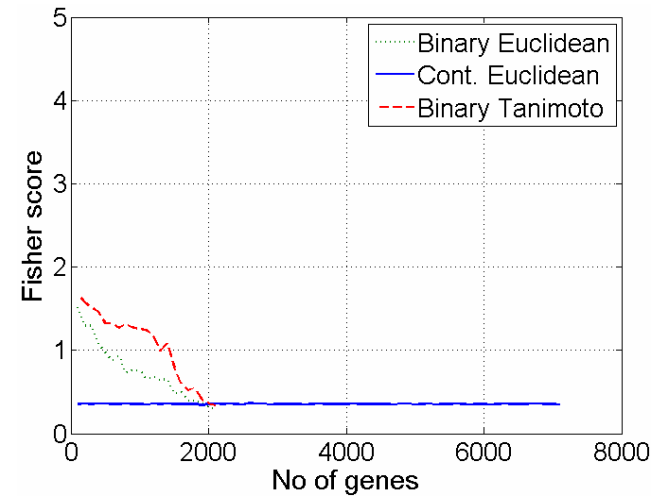

(c) )

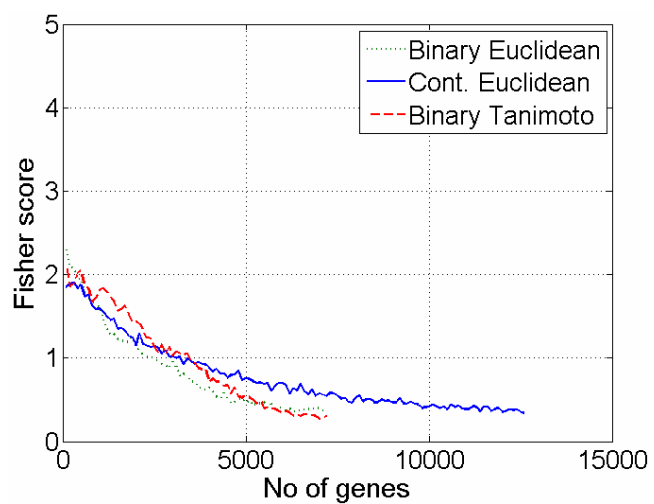

(b)

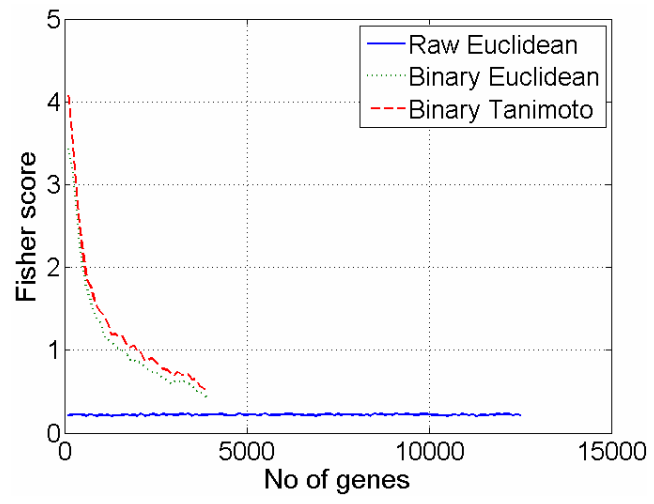

(d)

Figure 2. Comparison of spectral clustering results for four different datasets at various number of genes selected with Fisher Ratio. (a) is for [11], (b) is for [7], (c) is for [6] and (d) is for [8].

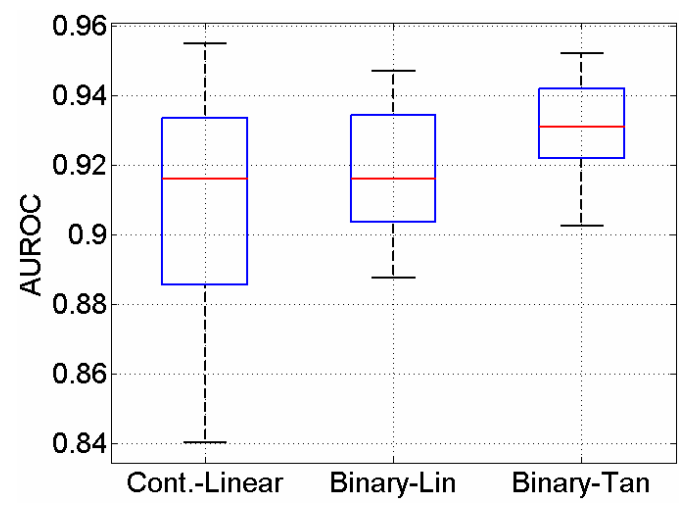

(a)

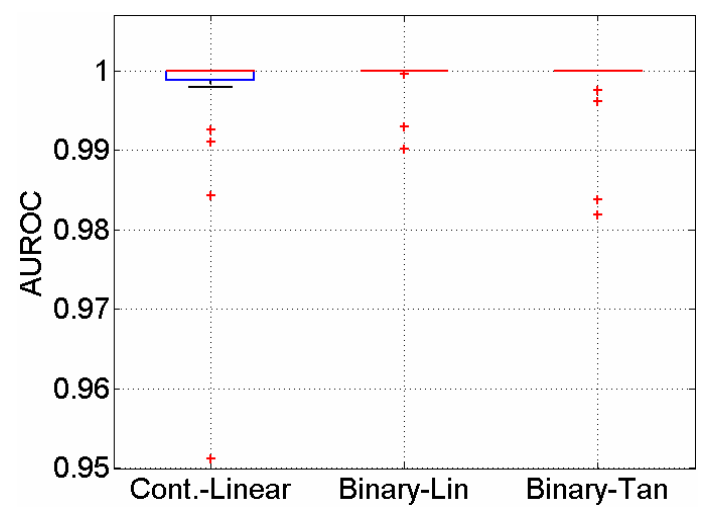

(b)

Figure 3. Reduction in variability of results due to preprocessing choice of algorithms. randomly chosen 38 combinations of preprocessing the CEL files produce large variations in classification results (leftmost columns). Working with discretized data reduces this variation in the inference. (a) data from [6], and (b) data from GSE2665.

\subsection{Reduction in Algorithmic Variability}

Figure 3 shows reduction in the variability caused by choice of preprocessing algorithms. Patterns of gene

\footnotetext{
${ }^{1}$ Also presented at the Microarray Gene Expression Society (MGED) meeting, Riva del Garda, Italy, September 2008.
}

expression levels change substantially with choice of algorithms, and this has a substantial effect on the resulting inference. A recent careful study (P. Boutros, personal communication ${ }^{1}$ ) established that this variability is significant. The leftmost columns of Figures 3(a) and (b) show this as box plots on two datasets. We see 
standard deviations in classifier performances, with outliers removed, of 0.032 and 0.134 respectively, and these reduce to 0.017 and 0.009 when the expression levels are binarized. The use of Tanimoto metric (box plots of the last columns of Figure 3) improves this even further.

\section{DATA AND METHODS}

\subsection{Approach}

Our approach was to show that on a sample of classification problems published in literature, classification accuracies reported by the authors do not significantly degrade when the gene expression data is quantized to binary precision (i.e. if the gene is expressed or not). Having achieved this, we implemented a similarity measure suitable for high dimensional binary spaces in a kernel framework to show that any loss of performance is easily recovered. In a number of cases the approach we took indeed produced better accuracies than working with the data at raw precision (see Results).

\subsection{Tanimoto Similarity}

Tanimoto coefficient ( $T$ ) [12], between two binary vectors, is defined as follow:

$$
T=\frac{c}{a+b-c}
$$

where

$a$ : the number of expressed points for gene $\mathrm{x}$,

$b$ : the number of expressed points in gene $\mathrm{y}$ and

$c$ : the number of common expressed points in two genes.

Tanimoto similarity ranges from 0 (no points in common) to 1 (exact match) [13] and is the rate of the number of common bits on to the total number of bits on two vectors. It focuses on the number of common bits that are on. The denominator of Tanimoto coefficient can be considered as a normalization factor which helps to reduce the bias of the vector size (i.e with larger vectors Tanimoto coefficients work better $[14,15]$. For this reason Tanimoto coefficient is the preferred similarity measure in chemoinformatics as all the vectors are long and there are only few bits on.

Tanimoto kernel can be defined as [16]:

$$
K_{\text {Tan }}(x, z)=\frac{x^{T} z}{x^{T} x+z^{T} z-x^{T} z}
$$

where $a=x^{T} x, \quad b=z^{T} z$ and $c=x^{T} z$. It follows from the work of Trotter [16] that this similarity metric satisfies Mercer conditions to be useful as a valid kernel: i.e. kernel computations in the space of the given binary vectors map onto inner products in a higher dimensional space so that SVM type optimizations for large margin class boundaries is possible.

Alternate ways of classification of binarized data can be considered. Motivated by the distance to barcode classifier built by Zilliox and Irizarry [2] we implemented similar classifiers. An obvious choice in these circumstances is to set two templates, one to represent each class, and position them at the centroids of the two class profiles. This is a distance to mean classifier in standard statistical pattern recognition terminology. A particular limitation of this strategy is discussed later. The barcodes designed by Zilliox and Irizarry [2], however, are not positioned at the centroids because they are evaluated by analysing a large number of archived experiments. We also built such discriminant templates, by doing a stochastic search starting from the centroids as initial condition. Such an optimization achieves templates that are better positioned in the input space than centroids for distance-based discrimination.

Clustering is the most popular tool in the analysis of microarray data. In order to conform whether the use of Tanimoto distance metric is useful in clustering, we applied the method of spectral clustering to the classification problems considered above. Without knowledge of the class labels, we clustered each of the datasets into two clusters using spectral clustering. Subsequently, using knowledge of the class labels we looked to see how well separated the clusters formed were, and how accurately the data was allocated to the right clusters. To measure cluster compactness we used the Fisher ratio as performance metric:

$$
\text { Fisher Score }=\frac{a b s\left(\mu_{1}-\mu_{2}\right)}{\sigma_{1}+\sigma_{2}}
$$

Checking if the examples were consistently associated with the right clusters, we computed percentage classification errors. The choice of classification problems to evaluate cluster compactness offers a far better setting than clustering genes into functions. This is because cluster analysis, when the data has large numbers of clusters in them, is notoriously unstable. With data taken from classification problems, we could expect well defined cluster formations (e.g. cancer versus non-cancer), in which we can compare the role of different distance metrics.

\subsection{Datasets}

We give a short description of the datasets used in our study.

- Yeast dataset compiled and first used in Brown et al. [9] for predicting yeast gene functions. cDNA arrays, in which the task is to classify 121 ribosomal genes from the remaining 2346 using 79 features. The features are hybridization conditions during cell cycle progression under different synchronization methods.

- Widely used Leukemia dataset (Golub et al., [11]); there are 5000 genes with 38 samples (27 
ALL, 11 AML), being the test subset of the full dataset.

- Colon dataset (Alon et al., [10]), 2000 genes with 62 samples (20 normal and 42 tumour samples).

- Two Breast cancer datasets, first one from (West et al., [6]) 7129 genes and 49 samples, (25 ER+ and 24 ER-) and the other Huang et al. [7] 12625 genes with 89 samples (depending on LN status).

- Lung cancer dataset, (Gordon et al., [8]), 12533 genes and 181 samples (31 malignant pleural mesothelioma (MPM) and 150 adenocarcinoma (ADCA)).

- 53 randomly selected datasets from ArrayExpress (http://www.ebi.ac.uk/arrayexpress/) and Gene Expression Omnibus (GEO)

(http://www.ncbi.nlm.nih.gov/geo/) for probe level uncertainty analysis analysis. Accession numbers of these datasets are:

GEO: GSE5666, GSE7041, GSE8000, GSE8505, GSE6487, GSE6850, GSE8238, GSE2665

Array Express: E-GEOD-6783, E-GEOD-6784\}, E-MEXP-1403, E-ATMX-30, E-GEOD-6647, E-GEOD-6620, E-ATMX-13, E-MEXP-1443, E-GEOD-2450, E-GEOD-2535, E-MEXP-914, E-MEXP-268, E-GEOD-2848, E-GEOD-2847, E-MEXP-430, E-GEOD-6321, E-MEXP-70, E-GEOD-1588, E-MEXP-727, E-TABM-291, E-GEOD-3076, E-GEOD-1938, E-GEOD-7763, E-GEOD-3854, E-GEOD-1639, E-TABM-169, E-MAXD-6, E-MEXP-526, E-GEOD-2343, E-GEOD-3846, E-MEXP-26, E-GEOD-1723, E-GEOD-1934, E-MAXD-6, E-MEXP-879, E-GEOD-10262, E-GEOD-10422, E-MEXP-998, E-MEXP-580, E-GEOD-10072, E-GEOD-10627.

\section{Web Pages:}

http://yeast.swmed.edu/cgi-bin/dload.cgi, http://data.genome.duke.edu/west.php, http://data.genome.duke.edu/lancet.php, http://chestsurg.org/publications/2002-microarray.aspx

- Synthetic data was produced following Dettling [17], using $\mathrm{R}$ code made available by the authors. Data is produced to follow the statistics (mean and correlation structure) of the leukaemia data [11]. We generated several realizations of 200 samples in 250 dimensions. We explored varying these values over a range, and results reported in this paper correspond to the above figures.

\subsection{Spectral Clustering}

Spectral clustering uses eigenvectors of the pairwise similarity matrix to partition the data. The most widely used distance metric to calculate the similarity matrix is the negative exponential of a scaled Euclidean distance.

$$
A_{(i, j)}=\exp \left(-\frac{\left\|x_{i}-x_{j}\right\|^{2}}{\sigma^{2}}\right)
$$

where the scale parameter $\sigma$ is a free tuning parameter. The steps involved in spectral clustering, in which we replace the similarity measure by Tanimoto similarity between binary strings, are summarized as follows:

- Pairwise similarity matrix $A_{i, j}$ between the genes $i$ and $j$ is calculated by using Tanimoto coefficient.

- Following Brewer [18] an exponential is applied:

$$
A_{i j}^{F}=\exp ^{-\alpha\left(A_{i j}-1\right)^{2}}
$$

- Compute the normalized Laplacian matrix.

$$
L=D^{-1 / 2} \times A^{F} \times D^{-1 / 2}
$$

- Compute the eigenvalue decomposition of $\mathrm{L}$.

$$
(D-L) y_{i}=\lambda_{i} D y_{i}
$$

- Select the eigenvector corresponding to the second smallest eigenvalue.

Parameters $\alpha$ and $\sigma$ were tuned by searching over a range of feasible values: $-5.0 \rightarrow 5.0$.

Uncertainties in results for cluster analysis were evaluated by a bootstrap method. For each of the tasks, 100 datasets of the same size as the original data were created by sampling with replacement before the application of the spectral clustering algorithm. Performances reported are averages and standard deviations across these 100 bootstrap samples.

\subsection{Optimised Templates}

The search to find templates better than class means for a distance-to-template classifier was implemented as a stochastic local search by means of a genetic algorithm. Templates were initialized to class means. At every step in an iterative search, we randomly changed $20 \%$ of the elements in the two templates, to derive mutated barcodes in their vicinity. Throughout the search, we retained ten best template pairs at any iteration. Large search steps were implemented by crossover operation between pairs of templates whereby half the bits in the patterns were swapped between pairs, a standard operation in genetic algorithms. We evaluated the accuracy of the resulting classifier and there was an improvement we retained the mutated templates, and discarded them if was no improvement. 


\subsection{Algorithmic Variability}

We used the EXPRESSO set of algorithms in package Affy in Bioconductor. For both datasets West et al. [6] and GSE2665, we worked from the CEL files and applied a total of 38 different preprocessing combinations from a total of 315 possibilities, randomly chosen.

\subsection{Other Details}

To analyse probe level uncertainties (Milo et al. [19]) we used the PUMA package (Propagating Uncertainty in Microarray Analysis), downloaded from the site (www.bioinf.manchester.ac.uk/resources/puma/). For quantization of microarray data, we used the method developed by Zhou et al. [20], which models gene expressions as mixture Gaussian densities. For quantization to binary levels, two Gaussians are used, resulting in two means and standard deviations: $\mu_{1}, \mu_{2}, \sigma_{1}$ and $\sigma_{2}$. From these a threshold $\theta$, is computed as $\theta=0.5 \times$ $\left(\mu_{1}+\mu_{2}+\sigma_{1}-\sigma_{2}\right)$. SVM implementations were done in the MATLAB SVM package described in Gunn [21] (http://www.isis.ecs.soton.ac.uk/isystems/kernel/).

\section{CONCLUSIONS}

The results suggest that a binary representation for transcriptomic data is indeed suitable and good classification accuracies can be obtained in this space using suitable similarity metrics cast in a kernel framework. There are two reasons for the superior performance of TanimotoSVM based approach over the distance to template approach inspired by the barcode approach.

\subsection{Distance to Template Classifier}

Why did the distance to template method not perform well consistently in classification problems? We suggest this result is largely to be expected. With continuous data, it is a well known result of statistical pattern recognition that classification by computing distances to a template is optimal only in the case that the distributions of each class is Gaussian, isotropic (i.e. variances of each feature is the same) and these variances are the same for both [22]. When any of these assumptions is violated, a distance to template classifier is no longer optimal. Even under the mild relaxations of the assumption, that of Gaussian densities with identical but nonisotropic covariance matrices, the optimal classifier requires computation of second order statistics in the form of the Mahalanobis distance to class means. In gene expression data isotropic variation cannot be assumed. Under regulation by combinatorial transcription factor activity where each transcription factor may control several genes, correlated expression of groups of genes should be expected. Indeed, the wide use of cluster analysis of microarray data is based on the assumption that correlated expression profiles might suggest co-regulation. Therefore, as uncorrelated features cannot be assumed, optimal classification is unlikely to be achieved by distance to template decision rules.

Does the same difficulty arise in the barcode method proposed by Zilliox and Irizarry (2007)? To verify this we took three datasets, one of which was not included in their analysis. Prediction accuracies for these three, comparing the barcode method to Tanimoto-SVM, are shown in Table 3. We note that training and testing on the same database, as we have done with Tanimoto-SVM, achieves consistently better prediction accuracies than the barcode method. But in fairness to the barcode method we remark that their intention is to make predictions on a new dataset based on accumulated historic knowledge, rather than repeat the training/testing process all over again. On this point, while there is impressive performance reported on the datasets Zilliox and Irizarry (2007). worked on, the method can fail badly too, as in the case of the lung cancer prediction task E-GEOD-10072 shown in Table 3.

Table 3. Comparison of Tanimoto-SVM with [2]'s barcode.

\begin{tabular}{cccc}
\hline Dataset & Data type & Method & Accuracy \\
\hline E-GEOD-10072 & Binary & Barcode & 0.50 \\
Lung & Binary & Tanimoto-SVM & $0.89 \pm 0.03$ \\
Lung tumor vs. normal & Binary & Tanimoto-SVM & $0.99 \pm 0.03$ \\
GSE2665 & Binary & Barcode & 0.95 \\
Lymph node/tonsil & Binary & Tanimoto-SVM & $0.99 \pm 0.02$ \\
lymph node vs. tonsil & Binary & Tanimoto-SVM & $1.0 \pm 0.0$ \\
GSE2603 & Binary & Barcode & 0.90 \\
Breast Tumor & Binary & Tanimoto-SVM & $0.99 \pm 0.01$ \\
Breast Tumor vs. normal & Binary & Tanimoto-SVM & $0.99 \pm 0.01$ \\
\hline
\end{tabular}




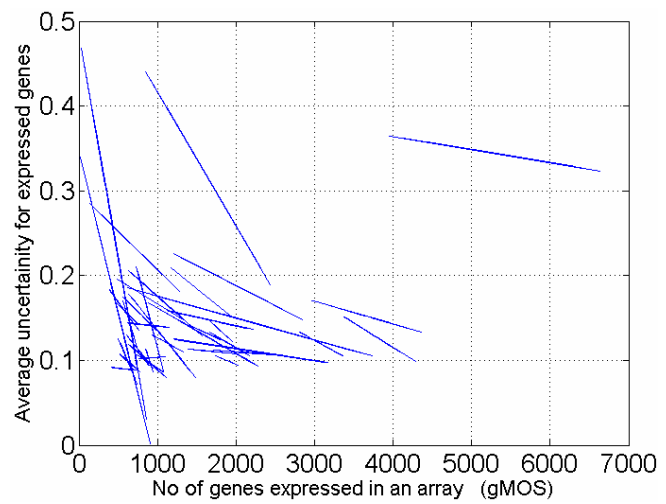

(a)

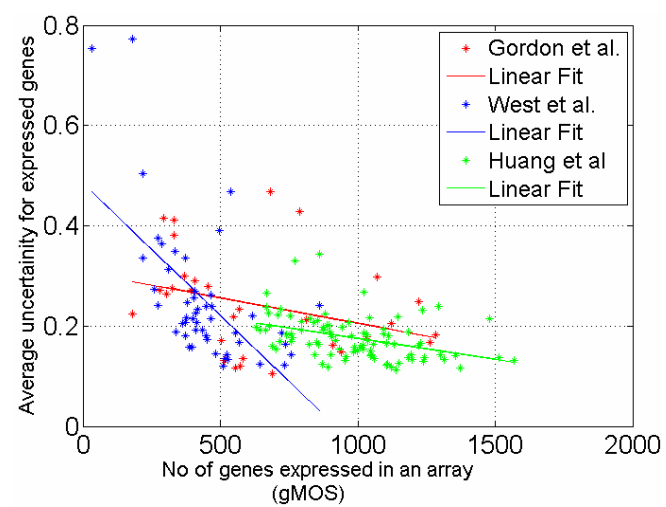

(b)

Figure 4. A systematic variation in probe level uncertainty of Affymetrix microarray data. (a) On 53 randomly chosen arrays we plot the average uncertainty of determining expression levels against the number of genes detected as present. Only liner regression lines are shown for clarity. (b) Scatter plots of uncertainties against number of expressed genes, and the linear regression lines, for the three datasets analysed in this paper.

\subsection{Probe Level Uncertainty}

The Tanimoto similarity metric attaches higher scores to profiles with large numbers of expressed genes. For example if we consider two pairs of vectors with

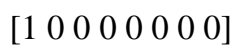

$$
\begin{aligned}
& {\left[\begin{array}{llllllll}
1 & 1 & 0 & 0 & 0 & 0 & 0 & 0
\end{array}\right] \text {, }} \\
& {\left[\begin{array}{llllllll}
1 & 1 & 0 & 0 & 0 & 0 & 0 & 0
\end{array}\right]}
\end{aligned}
$$

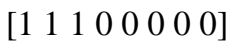

In both cases Hamming distance, thus Euclidean distance, is one. The Tanimoto similarities between these pairs, however, are different: 0.5 for the first pair and 0.66 for the second. We suggest that a reason why such a weighting on the similarity scores translates to improve clustering and class prediction performance comes from the uncertainties associated with microarray measurements. We found a systematic variation in uncertainties in expression levels as function of the numbers of expressed genes in an array. To illustrate this we used a probabilistic model of encapsulating probe level uncertainties introduced in Milo et al. (2003) [19], and plotted the average uncertainty in expressed genes as a function of the number of genes marked as expressed under our quantization scheme for several arbitrarily chosen datasets.

Figure 4 shows the variation in uncertainty with numbers of expressed genes, for three of the datasets on which we report classification results, and for 50 arbitrarily taken datasets from archives. We see that there is a systematic reduction in probe level uncertainty as the number of expressed genes in an array gets larger ${ }^{2}$. We offer no molecular level explanation for this, but the

\footnotetext{
${ }^{2}$ We stress that this variation is not a consequence of amplifying noise in the data of normalised poor quality arrays; i.e. for an array scanned at low intensity, normalization amplifies noise; the effect of such noise would be to increase the average uncertainty when more and more genes are taken as expressed. This is precisely the opposite of what we see in Figure 4.
}

effect is systematic and its impact on the Tanimoto-SVM is clear. Arrays with larger numbers of expressed genes are being measured with higher levels of confidence. Hence if we were to increase the weighting given to similarities between such profiles we would expect increased performance. Such probe level uncertainty has been of interest to other researchers, too. Rattray et al. [23] and Sanguinetti et al. [24] show how cluster analysis and visualization in a subspace by principal component projections can be carried out incorporating probe level uncertainty. In general these are errors-in-variables type models. We believe accounting for probe level (and other low level) uncertainties in microarray analysis is an important topic, and the systematic variability we have noted here may well be an aspect that other researchers can exploit in microarray inference.

\section{REFERENCES}

[1] I. Shmulevich and W. Zhang, (2002) Binary analysis and optimization-based normalization of gene expression data, Bioinformatics, 18(4), 555-565.

[2] M. J. Zilliox and R. A. Irizarry, (2007) A gene expression bar code for microarray data, Nature Methods, 4(11), 911-913.

[3] S. Tuna and M. Niranjan, (2009) Inference from low precision transcriptome data representation, Journal of Signal Processing Systems, [Online, 22 April 2009], doi: 10.1007/s11265-009-0363-2.

[4] S. Draghici, P. Khatri, A. C. Eklund, and Z. Szallasi, (2006) Reliability and reproducibility issues in DNA microarray measurements, Trends in Genetics, 22(2), 101109.

[5] D. Geman, C. d'Avignon, D. Q. Naiman, and R. L. Winslow, (2004) Classifying gene expression profiles from pairwise mRNA comparisons, Statistical Applications in Genetics and Molecular Biology, 3.

[6] M. West, C. Blanchette, H. Dressman, E. Huang, S. Ishida, R. Spang, H. Zuzan, J. A. Olson, J. R. Marks, and 
J. R. Nevins, (2001) Predicting the clinical status of human breast cancer by using gene expression profiles Proceedings of National Academy of Sciences, 98(20), 11462-11467.

[7] E. Huang, S. H. Cheng, H. Dressman, J. Pittman, M. Tsou, C. Horng, A. Bild, E. S. Iversen, M. Liao, C. Chen, M. West, J. R. Nevins, and A. T. Huang, (2003) Gene expression predictors of breast cancer outcomes Lancet, 361, 1590-1596.

[8] G. J. Gordon, R. V. Jensen, L. Hsiao, S. R. Gullans, J. E. Blumenstock, S. Ramaswamy, W. G. Richards, D. J. Sugarbaker, and R. Bueno, (2002) Translation of microarray data into clinically relevant cancer diagnostic tests using gene expression ratios in lung cancer and mesothelioma, Cancer Research, 62(17), 4963-4967.

[9] M. P. S. Brown, W. N. Grundy, D. Lin, N. Cristianini, C. W. Sugnet, T. S. Furey, M. Ares, and D. Haussler, (2000) Knowledge-based analysis of microarray gene expression data by using support vector machines, Proceedings of National Academy of Sciences, 97(1), 262-267.

[10] U. Alon, N. Barkai, D. A. Notterman, K. Gish, S. Ybarra, D. Mack, and A. J. Levine, (1999) Broad patterns of gene expression revealed by clustering analysis of tumor and normal colon tissues probed by oligonucleotide arrays, Proceedings of National Academy of Sciences, 96(12), 6745-6750.

[11] T. R. Golub, D. K. Slonim, P. Tamayo, C. Huard, M. Gaasenbeek, J. P. Mesirov, H. Coller, M. L. Loh, J. R. Downing, M. A. Caligiuri, C. D. Bloomfield, and E. S. Lander, (1999) Molecular classification of cancer: Class discovery and class prediction by gene expression monitoring, Science, 286(5439), 531-537.

[12] T. T. Tanimoto, (1958) "An elementary mathematical theory of classification and prediction,” IBM Internal Report.

[13] P. Willett, (2006) Similarity-based virtual screening using 2d fingerprints, Drug Discovery Today, 11(23/24), 1046-
1053.

[14] P. Willett, J. M. Barnard, and G. M. Downs, (1998) Chemical similarity searching, Journal of Chemical Information and Computer Sciences, 38(6), 983-996.

[15] J. D. Holliday, N. Salim, M. Whittle, and P. Willett, (2003) Analysis and display of the size dependence of chemical similarity coefficients, Journal of Chemical Information and Computer Sciences, 43(3), 819-828.

[16] M. Trotter, (2006) Support vector machines for drug discovery. PhD thesis, University College London, UK.

[17] M. Dettling, (2004) BagBoosting for tumor classification with gene expression data, Bioinformatics, 20(18), 35833593.

[18] M. Brewer, (2007) Development of a spectral clustering method for the analysis of molecular data sets, Journal of Chemical Information and Modeling, 47(5), 1727-1733.

[19] M. Milo, A. Fazeli, M. Niranjan, and N. D. Lawrence, (2003) A probabilistic model for the extraction of expression levels from oligonucleotide arrays, Biochemical Society Transactions, 31(6), 1510-1512.

[20] X. Zhou, X. Wang, and E. R. Dougherty, (2003) Binarization of microarray data on the basis of a mixture model, Molecular Cancer the Rapeutics, 2(7), 679-684.

[21] S. Gunn, (1998) Support vector machines for classification and regression, Tech. Rep., University of Southampton.

[22] R. O. Duda, P. E. Hart, and D. G. Stork, (2001) Pattern Classification, John Wiley \& Sons, USA, ISBN 0-4105669-3.

[23] M. Rattray, X. Liu, G. Sanguinetti, M. Milo, and N. Lawrence, (2006) Propagating uncertainty in microarray data analysis, Briefings in Bioinformatics, 7(1), 37-47.

[24] G. Sanguinetti, M. Milo, M. Rattray, and N. D. Lawrence, (2005) Accounting for probe-level noise in principal component analysis of microarray data, Bioinformatics, 21(19), 3748-3754. 